\title{
Numerical study for the BVP of the liquid film flow over an unsteady stretching sheet with thermal radiation and magnetic field
}

\author{
M.M. Khader ${ }^{1,2^{*}}$
}

\section{"Correspondence:}

mohamed.khader@fsc.bu.edu.eg; mohamedmbd@yahoo.com

'Department of Mathematics and

Statistics, College of Science,

Al Imam Mohammad Ibn Saud

Islamic University (IMSIU), Riyadh,

Saudi Arabia

${ }^{2}$ Department of Mathematics, Faculty of Science, Benha University, Benha, Egypt

\section{照 Springer}

\begin{abstract}
In this paper, we introduce a method based on replacement of the unknown function by truncated series of the well-known shifted Chebyshev (of third-kind) expansion of functions. We give an approximate formula for the integer derivative of this expansion. We state and prove some theorems on the convergence analysis. By means of collocation points the introduced method converts the proposed problem to solving a system of algebraic equations with shifted Chebyshev coefficients. As an application for this efficient numerical method, we employ it in solving the system of ordinary differential equation that describes the thin film flow and heat transfer with the effect of thermal radiation, magnetic field, and slip velocity.
\end{abstract}

MSC: $41 \mathrm{~A} 04 ; 65 \mathrm{~N} 12 ; 76502$

Keywords: Liquid film; Thermal radiation; Unsteady stretching sheet; Chebyshev collocation method; Convergence analysis

\section{Introduction}

The thin film fluid flow has become dependent on many theoretical and experimental studies in recent years due to its widespread applications in industry and engineering such as continuous casting, crystal growing, tinning of copper wires, chemical processing equipment, and wire and fiber coating. Many authors studied the thin film fluid flow and heat transfer under different cases; see, for example, [1-10]. In [11], the authors studied the flow of an incompressible liquid film down a wavy incline and applied the Galerkin method with only one ansatz function to the Navier-Stokes equations. They derived a second-order weighted residual integral boundary layer equation to describe eddies in the troughs of the wavy bottom. Marin [12] considered a cylinder made of a microstretch thermoelastic material for which one plane end is subjected to plane boundary data varying harmonically in time. On the lateral surface and other bases, we have zero body force and heat supply. Finally, Melvin and Herman [13] presented algorithmic matters of a computer code to solve linear two-point boundary-value problems. The proposed method used a superposition coupled with an orthonormalization procedure and a variable-step RungeKutta-Fehlberg integration scheme. Just and Stempien [14] studied the Pareto optimal control system for a nonlinear one-dimensional extensible beam equation and its Galerkin approximation. In [15], the authors proposed a modified and simple algorithm for frac-

(c) The Author(s) 2018. This article is distributed under the terms of the Creative Commons Attribution 4.0 International License (http://creativecommons.org/licenses/by/4.0/), which permits unrestricted use, distribution, and reproduction in any medium, provided you give appropriate credit to the original author(s) and the source, provide a link to the Creative Commons license, and indicate if changes were made. 
tional modeling arising in unidirectional propagation of long wave in dispersive media by using the fractional homotopy analysis transform method. The proposed technique can be used to solve nonlinear problems without using the Adomian and He's polynomials, which can be considered as a clear advantage of this new algorithm over decomposition and the homotopy perturbation transform method. This modified method yields an analytical and approximate solution in terms of a rapidly convergent series with easily computable terms. Also, exploiting variational methods and the existence of multiple weak solutions for a class of elliptic Navier boundary problems involving the $p$-biharmonic operator are investigated in [16]. Finally, the radiative effects for some bidimensional thermoelectric problems are investigated in [17].

After these previous publications, a number of researchers have successfully applied several numerical methods in this field. Among these numerical methods, the Chebyshev collocation method is a general approximate analytical method used to get the solutions for some of nonlinear differential equations. The Chebyshev collocation method has some advantages for handling this class of problems, in which the Chebyshev coefficients for the solution can be calculated very easily by numerical programs. For this reason, this method is much faster than the other methods. Chebyshev polynomials are a well-known family of orthogonal polynomials on the interval $[-1,1]$ with many applications. They are widely used because of their good properties in the approximation of functions [18-20]. Some of these properties take a very concise form in the case of the Chebyshev polynomials, making them of leading importance among orthogonal polynomials. The Chebyshev polynomials belong to an exclusive class of orthogonal polynomials, known as Jacobi polynomials, which correspond to weight functions of the form $(1-x)^{\alpha}(1+x)^{\beta}$ and which are solutions of Sturm-Liouville equations. The Chebyshev collocation method is used to solve many problems in many papers, for example, [18-20].

In this work, we use the properties of Chebyshev polynomials to derive an approximate formula of the integer derivative of the approximate solution and estimate an error upper bound of this formula. Due to a high accuracy of this method, it is inevitable to use it to solve numerically the resulting nonlinear system of ordinary differential equations, which describe a flow and heat transfer of thin liquid film affected by the presence of thermal radiation and magnetic field.

The basic ordinary differential equations that describe the thin film liquid layer flow and heat transfer can be summarized as follows [21]:

$$
\begin{aligned}
& f^{\prime \prime \prime}+\gamma\left[f f^{\prime \prime}-S \frac{\eta}{2} f^{\prime \prime}-f^{2}-(S+M) f^{\prime}\right]=0, \\
& \frac{1}{\operatorname{Pr}}(1+R) \theta^{\prime \prime}+\gamma\left[f \theta^{\prime}-2 f^{\prime} \theta-\frac{S}{2}\left[3 \theta+\eta \theta^{\prime}\right]\right]=0, \\
& f(0)=0, \quad f(1)=\frac{1}{2} S, \quad \theta(0)=1, \\
& f^{\prime \prime}(1)=0, \quad f^{\prime}(0)=1+\delta f^{\prime \prime}(0), \quad \theta^{\prime}(1)=0,
\end{aligned}
$$

where the prime denotes differentiation with respect to $\eta, S$ is the unsteadiness parameter, $M$ is the magnetic parameter, $R$ is the radiation parameter, $\delta$ is the slip velocity parameter, $\gamma$ is the dimensionless film thickness, and $\operatorname{Pr}$ is the Prandtl number. Here, we must refer 
that the previous system of equations is a generalization of the pioneering research of Wang [21]. Our problem can be reduced to the Wang problem by taking $M=R=\delta=0$.

\section{Procedure of solution}

\subsection{Approximate the solution and its convergence analysis}

The Chebyshev polynomials of the third kind of degree $n$ are defined as

$$
\operatorname{Ch}_{n}(x)=\frac{\cos ((n+0.5) \varphi)}{\cos (0.5 \varphi)}, \quad x=\cos (\varphi), \quad 0 \leq \varphi \leq \pi .
$$

It well known that this family of polynomials is orthogonal on the interval $[-1,1]$ with respect to the weight function $w(x)=\sqrt{(1+x) /(1-x)}$ :

$$
\int_{-1}^{1} w(x) \operatorname{Ch} 3_{r}(x) \operatorname{Ch} 3_{s}(x) d x= \begin{cases}0 & \text { if } r \neq s \\ \pi & \text { if } r=s\end{cases}
$$

These polynomials are widely used because of their good properties in the approximation of functions and can be determined by the following recurrence formula [22]:

$$
\begin{aligned}
& \operatorname{Ch}_{3_{n+1}}(x)=2 x \operatorname{Ch} 3_{n}(x)-\operatorname{Ch} 3_{n-1}(x), \quad \operatorname{Ch} 3_{0}(x)=1, \\
& \operatorname{Ch} 3_{1}(x)=2 x-1, \quad n=1,2, \ldots .
\end{aligned}
$$

In this paper, we use these polynomials on the interval $[0,1]$, that is, the so-called shifted Chebyshev polynomials by introducing the change of variable $x=2 t-1, \overline{\mathbb{T}}_{n}(t)=\mathrm{Ch} 3_{n}(2 t-$ 1 ), where $\overline{\mathbb{T}}_{0}(t)=1, \overline{\mathbb{T}}_{1}(t)=4 t-3$. The most used and useful formula of $\overline{\mathbb{T}}_{n}(t)$ is given analytically:

$$
\overline{\mathbb{T}}_{n}(t)=\sum_{k=0}^{n}(-1)^{k} 2^{2 n-2 k} \frac{(2 n+1) \Gamma(2 n-k+1)}{\Gamma(k+1) \Gamma(2 n-2 k+2)} t^{n-k}, \quad n=2,3, \ldots .
$$

Every function $\Omega(t) \in L_{2}[0,1]$ can be expressed as a linear combination of $\left\{\overline{\mathbb{T}}_{i}(t)\right\}_{i=0}^{\infty}$ as

$$
\Omega(t)=\sum_{i=0}^{\infty} a_{i} \overline{\mathbb{T}}_{i}(t)
$$

with the coefficients $a_{i}, i=0,1, \ldots$, given by

$$
a_{i}=\frac{2}{\pi} \int_{0}^{1} \bar{w}(t) \Omega(t) \overline{\mathbb{T}}_{i}(t) d t, \quad i=0,1, \ldots
$$

where $\bar{w}(t)=\sqrt{\frac{t}{1-t}}$ is the shifted weight function.

By taking the first $m+1$ terms of (5) we obtain the following approximation:

$$
\Omega_{m}(t)=\sum_{i=0}^{m} a_{i} \overline{\mathbb{T}}_{i}(t)
$$

Theorem 1 Suppose that the function $\Omega(t)$ satisfies the following conditions: 
1. The second derivative $\Omega^{\prime \prime}(t)$ is a square-integrable function on $[0,1]$, i.e., $\Omega^{\prime \prime}(t) \in L_{2}[0,1]$

2. The second derivative $\Omega^{\prime \prime}(t)$ is bounded on $[0,1]$, i.e., $\left|\Omega^{\prime \prime}(t)\right| \leq \ell$ for some constant $\ell$.

Then the infinite series (5) of the shifted Chebyshev expansion is uniformly convergent, and

$$
\left|a_{i}\right|<\ell / i^{2}, \quad i=1,2, \ldots
$$

Proof We will use the change of variables in the integration of formula (6) for $a_{i}$ by using the transformation $2 t-1=\cos (\varphi)$ to obtain

$$
a_{i}=\frac{2}{\pi} \int_{0}^{\pi} y(0.5(1+\cos (\varphi))) \cos ((i+0.5) \varphi) \cos (0.5 \varphi) d \varphi .
$$

Now, using twice integration by parts and simple calculations, we find

$$
a_{i}=\frac{1}{4 \pi} \int_{0}^{\pi} \Omega^{\prime \prime}(0.5(1+\cos (\varphi))) \rho_{i}(\varphi) d \varphi
$$

where

$$
\begin{aligned}
\rho_{i}(\varphi) & =\sin (\varphi)\left[\frac{1}{i}\left(\frac{\sin (i-1) \varphi}{i-1}-\frac{\sin (i+1) \varphi}{i+1}\right)+\frac{1}{i+1}\left(\frac{\sin (i) \varphi}{i}-\frac{\sin (i+2) \varphi}{i+2}\right)\right], \\
\left|c_{i}\right| & =\left|\frac{1}{4 \pi} \int_{0}^{\pi} \Omega^{\prime \prime}(0.5(1+\cos (\varphi))) \rho_{i}(\varphi) d \varphi\right| \\
& \leq \frac{\ell}{4 \pi} \int_{0}^{\pi}\left|\rho_{i}(\varphi)\right| d \varphi \\
& \leq \frac{\ell}{4}\left[\frac{1}{i}\left(\frac{1}{i-1}+\frac{1}{i+1}\right)+\frac{1}{i+1}\left(\frac{1}{i}+\frac{1}{i+2}\right)\right] \\
& =\frac{\ell\left(2 i^{2}+2 i-1\right)}{2(i-1) i(i+1)(i+2)} .
\end{aligned}
$$

Then, for large $i$, we can get the required formula (8), and hence it uniformly converges to $\Omega(t)$, which completes the proof.

Theorem 2 Suppose that $\Omega(t) \in C^{m}[0,1]$. Then the error $E_{m}=\left\|\Omega(t)-\Omega_{m}(t)\right\|$ of approximation of the function $\Omega(t)$ by $\Omega_{m}(t)$ using formula (7) can be estimated as follows:

$$
\left\|\Omega(t)-\Omega_{m}(t)\right\| \leq \frac{\hbar \Delta^{m+1}}{(m+1) !} \sqrt{\frac{\pi}{2}}
$$

where $\hbar=\max _{t \in[0,1]} \Omega^{(m+1)}(t)$ and $\Delta=\max \left[t_{0}, t-t_{0}\right]$, and $C^{m}[0,1]$ is the space of all $m$ times continuously differentiable functions in the interval $[0,1]$.

Proof We will consider the Taylor polynomial $T_{m}(t)$ of degree $m$ defined by

$$
T_{m}(t)=\sum_{k=0}^{m} \Omega^{(k)}\left(t_{0}\right) \frac{\left(t-t_{0}\right)^{k}}{k !}
$$

as an approximation of the function $\Omega(t)$, where $t_{0} \in[0,1]$. 
We know that the error of this approximation is defined by

$$
\left|\Omega(t)-T_{m}(t)\right|=\left|\Omega^{(m+1)}(\tau) \frac{\left(t-t_{0}\right)^{m+1}}{(m+1) !}\right|, \quad \tau \in\left[t_{0}, t\right] .
$$

Now, from these approximations and some facts we can estimate

$$
\begin{aligned}
\left\|\Omega(t)-\Omega_{m}(t)\right\|^{2} & \leq\left\|\Omega(t)-T_{m}(t)\right\|^{2} \\
& =\int_{0}^{1} \bar{w}(t)\left[\Omega(t)-T_{m}(t)\right]^{2} d t \\
& =\int_{0}^{1} \bar{w}(t)\left[\Omega^{(m+1)}(\tau) \frac{\left(t-t_{0}\right)^{m+1}}{(m+1) !}\right]^{2} d t \\
& \leq \frac{\hbar^{2}}{[(m+1) !]^{2}} \int_{0}^{1} \bar{w}(t)\left(t-t_{0}\right)^{2 m+2} d t \\
& =\frac{\hbar^{2}}{[(m+1) !]^{2}} \int_{0}^{1}\left(t-t_{0}\right)^{2 m+2} \sqrt{\frac{t}{1-t}} d t .
\end{aligned}
$$

Then, denoting $\Delta=\max \left[t_{0}, t-t_{0}\right]$, we have

$$
\begin{aligned}
\left\|\Omega(t)-\Omega_{m}(t)\right\|^{2} & \leq \frac{\hbar^{2} \Delta^{2 m+2}}{[(m+1) !]^{2}} \int_{0}^{1} \sqrt{\frac{t}{1-t}} d t \\
& =\frac{\hbar^{2} \Delta^{2 m+2}}{[(m+1) !]^{2}} \frac{\pi}{2}
\end{aligned}
$$

Taking the square roots of both sides, we can get the required formula (9), which completes the proof.

In the following theorem,we give the main approximate formula for the integer derivative $D^{(n)} \Omega_{m}(t)$.

Theorem $3([23,24])$ Suppose that we approximate the function $\Omega(t)$ in the form $(7)$. Then $D^{(n)}\left(\Omega_{m}(t)\right)$ can be defined as

$$
D^{(n)}\left(\Omega_{m}(t)\right)=\sum_{i=n}^{m} \sum_{k=0}^{i-n} a_{i} \pi_{i, k}^{(n)} t^{i-k-n}, \quad \pi_{i, k}^{(n)}=\frac{(-1)^{k} 2^{2 i-2 k}(2 n+1)(2 i-k) !(i-k) !}{(k !)(2 i-2 k+1) !(i-k-n) !} .
$$

Proof The proof of this theorem can be done directly with the help of formula (7) and some properties of the third-kind shifted Chebyshev polynomials.

\subsection{Procedure solution}

In this section, we propose a method to solve numerically the system of ordinary differential equations of the form (1)-(2). The unknown functions $f(\eta)$ and $\theta(\eta)$ may be expanded by finite series of shifted Chebyshev polynomials as

$$
f_{m}(\eta)=\sum_{k=0}^{m} f_{k} \overline{\mathbb{L}}_{k}(\eta), \quad \theta_{m}(\eta)=\sum_{k=0}^{m} \theta_{k} \overline{\mathbb{L}}_{k}(\eta)
$$


From Eqs. (1)-(2) and (12) and formula (11) we have

$$
\begin{aligned}
& \sum_{i=3}^{m} \sum_{k=3}^{i-3} f_{i} \pi_{i, k}^{(3)} \eta^{i-k-3} \\
&+ \gamma\left[\left(\sum_{i=0}^{m} f_{i} \overline{\mathbb{L}}_{i}(\eta)\right)\left(\sum_{i=2}^{m} \sum_{k=2}^{i-2} f_{i} \pi_{i, k}^{(2)} \eta^{i-k-2}\right)-\frac{S}{2} \eta\left(\sum_{i=2}^{m} \sum_{k=2}^{i-2} f_{i} \pi_{i, k}^{(2)} \eta^{i-k-2}\right)\right. \\
&\left.-\left(\sum_{i=1}^{m} \sum_{k=1}^{i-1} f_{i} \pi_{i, k}^{(1)} \eta^{i-k-1}\right)^{2}-(S+M)\left(\sum_{i=1}^{m} \sum_{k=1}^{i-1} f_{i} \pi_{i, k}^{(1)} \eta^{i-k-1}\right)\right]=0, \\
& \frac{1+R}{m} \sum_{i=2}^{m} \sum_{k=2}^{i-2} \theta_{i} \pi_{i, k}^{(2)} \eta^{i-k-2}+\gamma\left[\left(\sum_{i=0}^{m} f_{i} \overline{\mathbb{L}}_{i}(\eta)\right)\left(\sum_{i=1}^{m} \sum_{k=1}^{i-1} \theta_{i} \pi_{i, k}^{(1)} \eta^{i-k-1}\right)\right. \\
&-2\left(\sum_{i=0}^{m} \theta_{i} \overline{\mathbb{L}}_{i}(\eta)\right)\left(\sum_{i=1}^{m} \sum_{k=1}^{i-1} f_{i} \pi_{i, k}^{(1)} \eta^{i-k-1}\right) \\
&-\frac{S}{2}\left[3\left(\sum_{i=0}^{m} \theta_{i} \overline{\mathbb{L}}_{i}(\eta)\right)+\eta\left(\sum_{i=1}^{m} \sum_{k=1}^{i-1} \theta_{i} \pi_{i, k}^{(1)} \eta^{i-k-1}\right)\right]=0 .
\end{aligned}
$$

We now collocate Eqs. (13)-(14) at $(m-n+1)$ points $\eta_{s}, s=0,1, \ldots, m-n$, as

$$
\begin{aligned}
\sum_{i=3}^{m} \sum_{k=3}^{i-3} f_{i} \pi_{i, k}^{(3)} \eta_{s}^{i-k-3} & {\left[\left(\sum_{i=0}^{m} f_{i} \overline{\mathbb{L}}_{i}\left(\eta_{s}\right)\right)\left(\sum_{i=2}^{m} \sum_{k=2}^{i-2} f_{i} \pi_{i, k}^{(2)} \eta_{s}^{i-k-2}\right)-\frac{S}{2} \eta_{s}\left(\sum_{i=2}^{m} \sum_{k=2}^{i-2} f_{i} \pi_{i, k}^{(2)} \eta_{s}^{i-k-2}\right)\right.} \\
+ & \gamma \\
- & \left.\left(\sum_{i=1}^{m} \sum_{k=1}^{i-1} f_{i} \pi_{i, k}^{(1)} \eta_{s}^{i-k-1}\right)^{2}-(S+M)\left(\sum_{i=1}^{m} \sum_{k=1}^{i-1} f_{i} \pi_{i, k}^{(1)} \eta_{s}^{i-k-1}\right)\right]=0, \\
\frac{1+R}{\operatorname{Pr}} & \sum_{i=2}^{m} \sum_{k=2}^{i-2} \theta_{i} \pi_{i, k}^{(2)} \eta_{s}^{i-k-2}+\gamma\left[\left(\sum_{i=0}^{m} f_{i} \overline{\mathbb{L}}_{i}\left(\eta_{s}\right)\right)\left(\sum_{i=1}^{m} \sum_{k=1}^{i-1} \theta_{i} \pi_{i, k}^{(1)} \eta_{s}^{i-k-1}\right)\right. \\
- & 2\left(\sum_{i=0}^{m} \theta_{i} \overline{\mathbb{L}}_{i}\left(\eta_{s}\right)\right)\left(\sum_{i=1}^{m} \sum_{k=1}^{i-1} f_{i} \pi_{i, k}^{(1)} \eta_{s}^{i-k-1}\right) \\
- & \frac{S}{2}\left[3\left(\sum_{i=0}^{m} \theta_{i} \overline{\mathbb{L}}_{i}\left(\eta_{s}\right)\right)+\eta_{s}\left(\sum_{i=1}^{m} \sum_{k=1}^{i-1} \theta_{i} \pi_{i, k}^{(1)} \eta_{s}^{i-k-1}\right)\right]=0 .
\end{aligned}
$$

For suitable collocation points, we use the roots of shifted Chebyshev polynomial $\overline{\mathbb{L}}_{m-n+1}(\eta)$. Also, by substituting formula (12) into the boundary conditions (3)-(4) we can obtain the following six equations:

$$
\begin{aligned}
& \sum_{i=0}^{m}(-1)^{i}(2 i+1) f_{i}=0, \quad \sum_{i=0}^{m} f_{i}=\frac{S}{2}, \quad \sum_{i=0}^{m}(-1)^{i}(2 i+1) \theta_{i}=1, \\
& \sum_{i=0}^{m} r_{i} f_{i}-\delta \sum_{i=0}^{m} \overline{\bar{r}}_{i} f_{i}=1, \quad \sum_{i=0}^{m} \overline{\bar{r}}_{i} f_{i}=0, \quad \sum_{i=0}^{m} \bar{r}_{i} \theta_{i}=0
\end{aligned}
$$


Table 1 Comparison of $\gamma$ and $-f^{\prime \prime}(0)$ with $\delta=M=0$ using the previous work and the Chebyshev collocation method

\begin{tabular}{llllll}
\hline$S$ & Data of [25] & & & Present results & \\
\cline { 2 - 3 } \cline { 5 - 6 } & $\gamma$ & & & & $\gamma$ \\
\hline 1.4 & 0.674089 & 1.012781 & & 0.6739267 & $-f^{\prime \prime}(0)$ \\
1.6 & 0.331976 & 0.642412 & & 0.309138 & 1.0126853 \\
1.8 & 0.127013 & 0.3320138 & & 0.1270089 & 0.6423921 \\
\hline
\end{tabular}

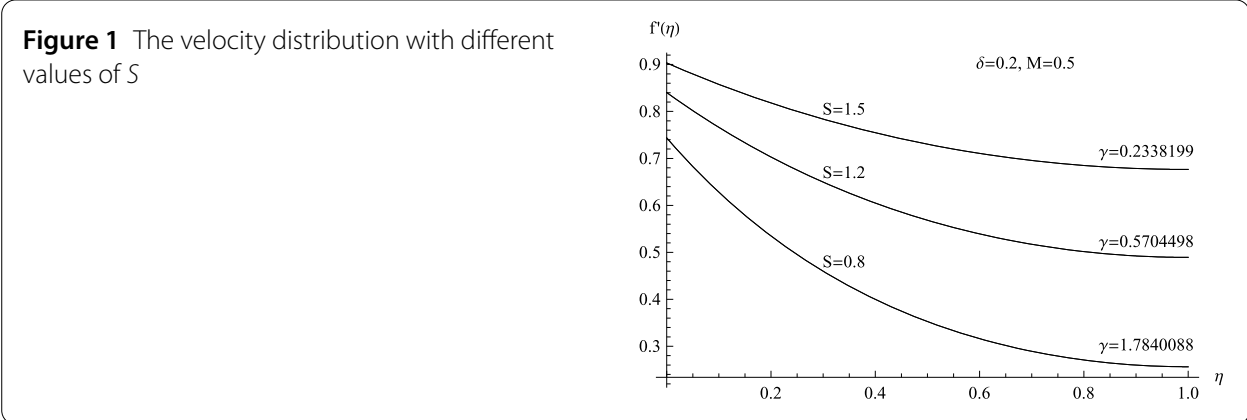

where $r_{i}=\overline{\mathbb{L}}_{i}^{\prime}(0), \bar{r}_{i}=\overline{\mathbb{L}}_{i}^{\prime}(1), \overline{\bar{r}}_{i}=\overline{\mathbb{L}}_{i}^{\prime \prime}(0), \overline{\bar{r}}_{i}=\overline{\mathbb{L}}_{i}^{\prime \prime}(1)$.

Equations (15)-(16), together with five equations of the boundary conditions (17), give a system of $(2 m+2)$ algebraic equations, which can be solved, for the unknowns $f_{i}, \theta_{i}, i=$ $0,1, \ldots, m$, using the Newton iteration method. In our numerical study, we take $m=5$, that is, five terms of the truncated series solution (12) at $\eta=1$.

\section{Results and discussion}

Firstly, to verify the accuracy of our numerical scheme, a comparison of the computed values of $-f^{\prime \prime}(0)$ is made by Noor and Hashim [25]. This comparison is illustrated in Table 1. Note that there is a close agreement between our data and the previous published work of [25], which lends confidence to the proposed numerical method.

Also, in this section, we discuss and analyze the solution of the described problem via figures that result from using the Chebyshev collocation method. Since the problems that arise in industrial and technological situations are highly nonlinear, it may be difficult to get analytical solutions to this type problems. One such problem, which occurs in the tinning of copper wires, polymer industry, and crystal growing, involves the thin flow of a Newtonian fluid past a stretching sheet. A relatively new method, called the shifted Chebyshev collocation, is very beneficial for developing solutions that are valid even for moderate to large values of parameters. This method depends on the series solution. One of the chief factors that influences the convergence of the solution series is the type of base functions used to express the solution. After applying the shifted Chebyshev collocation method, the solutions of the nonlinear system of ODEs (1)-(4) are presented through Figs. 1-7.

Figure 1 highlights the effect of $S$ on the dimensionless velocity $f^{\prime}(\eta)$. These plots reveal the fact that increasing values of the parameter $S$ results in enhancing the velocity distribution along the thin film region, but the reverse is observed for the film thickness. The dimensionless temperature for different values of unsteadiness parameter $S$ is displayed in Fig. 2. Note that both the free surface temperature $\theta(\gamma)$ and the dimensionless temperature increases with the increase in $S$. 


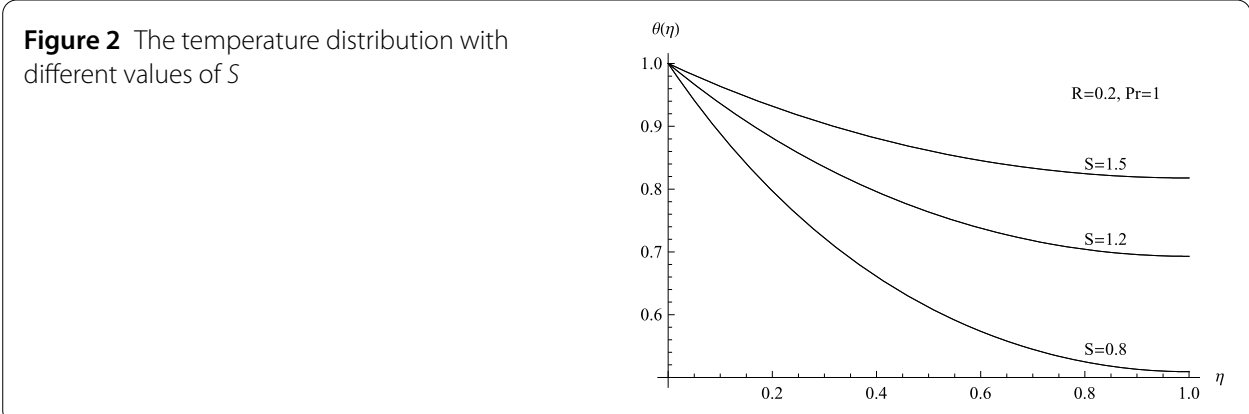

Figure 3 The velocity distribution with different values of $M$

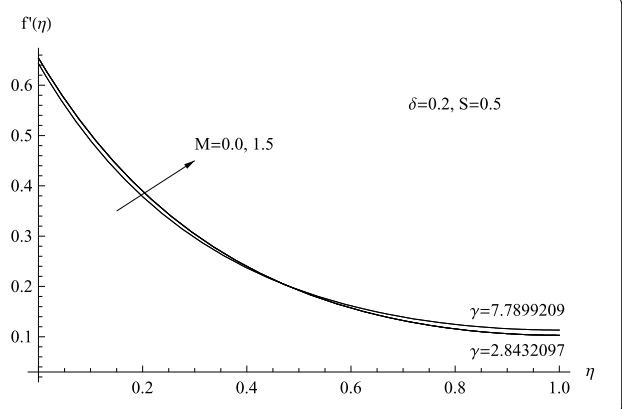

Figure 4 The temperature distribution with different values of $M$

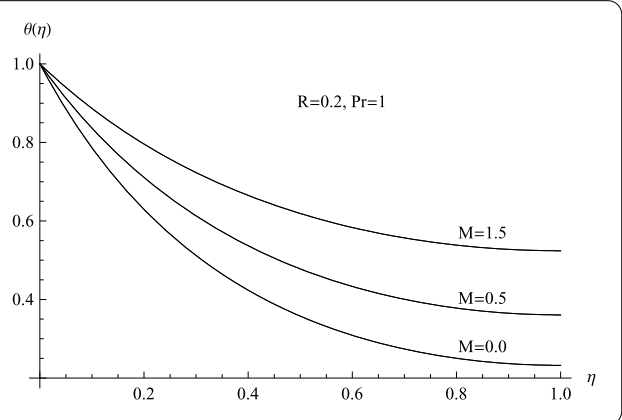

The influence of magnetic parameter $M$ on the dimensionless velocity is presented in Fig. 3. Observed that, along the sheet, the dimensionless velocity increases with an increase in the $M$ parameter, but the reverse trend is away from the sheet. Also, we further find that the increasing value of the parameter $M$ leads to decreasing the film thickness. In Fig. 4, we depict the effect of the same parameter $M$ on the dimensionless temperature. Note that, with the increasing values of magnetic parameter $M$, both the free surface temperature $\theta(\gamma)$ and the dimensionless temperature increase inside the film region.

Figure 5 represents the variation of velocity profiles for different values of the slip velocity parameter $\delta$. From this plot it is evident that, along the sheet, the effect of increasing values of the parameter $\delta$ is responsible for thinning the film thickness and decreasing the dimensionless velocity. Figure 6 displays the temperature $\theta(\eta)$ profiles versus $\eta$ for various values of the same parameter $\delta$. It is elucidating that both the dimensionless temperature distribution and the free surface temperature $\theta(\gamma)$ increase with an increase in parameter $\delta$. Figure 7 depicts the effect of the radiation parameter $R$ on the temperature profile. It is interesting to note that the dimensionless temperature distribution increases as the radiation parameter $R$ increases. Likewise, some qualitative behaviors for the thin film flow and 

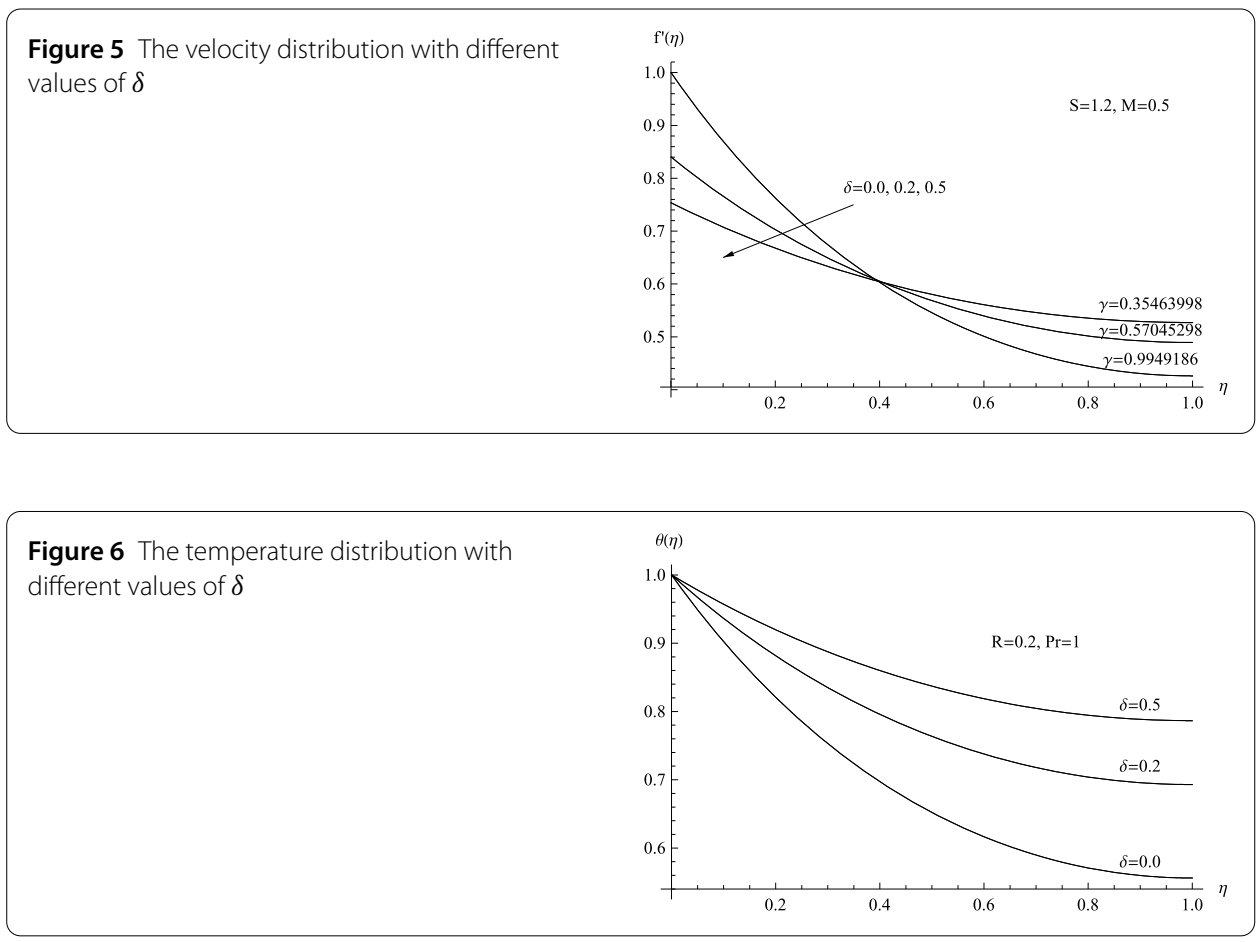

Figure 7 The temperature distribution with different values of $R$

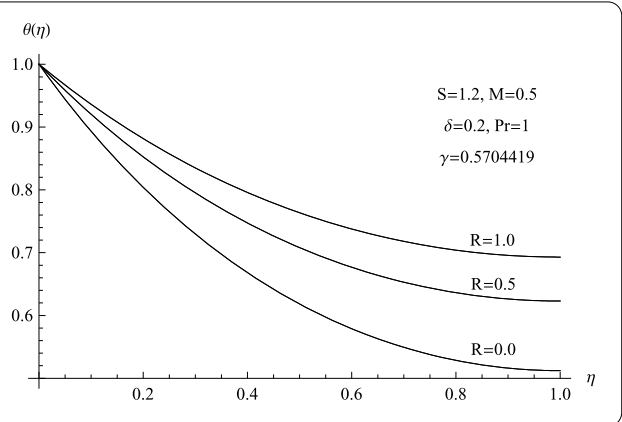

heat transfer characteristics are demonstrated in the same figure, in which the thickness for the film is fixed for changing the values of the radiation parameter $R$.

\section{Conclusion and remarks}

This numerical study highlights the lineaments of radiation parameter, velocity slip parameter, magnetic number, and the unsteadiness parameter in the flow and heat transfer for a Newtonian thin liquid film. We use the Chebyshev collocation method to investigate the approximate solution of the resulting nonlinear system of ODEs for the proposed problem. Numerical evaluations of the closed-form results are performed, and graphical results are obtained to illustrate the details of the thin film flow and heat transfer characteristics and their dependence on some physical parameters. On the basis of the obtained results, we can see the following observations:

(1) The effect of increasing both the values of the unsteadiness parameter and the magnetic parameter increases both the dimensionless velocity and the dimensionless temperature throughout the film layer. 
(2) The temperature distribution can be affected by changing the values of the velocity slip parameter and the thermal radiation parameter.

(3) Because of the presence of slip velocity parameter, there may be a lower velocity distribution near the stretching sheet and also thinning the film thickness.

\begin{abstract}
Acknowledgements
The author is very grateful to the editor and referees for carefully reading the paper and for their comments and suggestions, which have improved the paper.
\end{abstract}

Funding

Not applicable.

Availability of data and materials

Not applicable.

\title{
Competing interests
}

The author declares that there is no conflict of interests regarding the publication of this paper.

\section{Authors' contributions}

The paper by one author. Author read and approved the final manuscript.

\section{Publisher's Note}

Springer Nature remains neutral with regard to jurisdictional claims in published maps and institutional affiliations.

\section{Received: 31 December 2017 Accepted: 7 May 2018 Published online: 16 May 2018}

\section{References}

1. Wang, C.Y.: Liquid film on an unsteady stretching sheet. Q. Appl. Math. XLVIII, 601-610 (1990)

2. Usha, R., Sridharan, R.: The axisymmetric motion of a liquid film on an unsteady stretching surface. ASME Fluids Eng. $117,81-85$ (1995)

3. Andersson, H.I., Aarseth, J.B., Dandapat, B.S.: Heat transfer in a liquid film on an unsteady stretching surface. Int. J. Heat Mass Transf. 43, 69-74 (2000)

4. Dandapat, B.S., Santra, B., Andersson, H.I.: Thermocapillarity in a liquid film on an unsteady stretching surface. Int. J. Heat Mass Transf. 46, 3009-3015 (2003)

5. Dandapat, B.S., Maity, S.: Flow of a thin liquid film on an unsteady stretching sheet. Phys. Fluids 18, 102101 (2006)

6. Dandapat, B.S., Santra, B., Vajravelu, K.: The effects of variable fluid properties and thermocapillarity on the flow of a thin film on an unsteady stretching sheet. Int. J. Heat Mass Transf. 50, 991-996 (2007)

7. Liu, I.-C., Andersson, H.I.: Heat transfer in a liquid film on an unsteady stretching sheet. Int. J. Therm. Sci. 47, 766-772 (2008)

8. Santra, B., Dandapat, B.S.: Unsteady thin-film flow over a heated stretching sheet. Int. J. Heat Mass Transf. 52, 1965-1970 (2009)

9. Noor, N.F.M., Abdulaziz, O., Hashim, l.: MHD flow and heat transfer in a thin liquid film on an unsteady stretching sheet by the HAM. Int. J. Numer. Methods Fluids 63, 357-373 (2009)

10. Conte, S.D.: The numerical solution of linear boundary value problems. SIAM Rev. 8(3), 309-321 (1966)

11. Häcker, T., Uecker, H.: An integral boundary layer equation for film flow over inclined wavy bottoms. Phys. Fluids 21(9), 1-10 (2009)

12. Marin, M.: Harmonic vibrations in thermoelasticity of microstretch materials. J. Vib. Acoust. 132(4), 1-6 (2010)

13. Scott, M.R., Watts, H.A.: Computational solution of linear two-point boundary value problems via orthonormalization. SIAM J. Numer. Anal. 14(1), 40-70 (1977)

14. Just, A., Stempien, Z:: Pareto optimal control problem and its Galerkin approximation for a nonlinear one-dimensional extensible beam equation. Opusc. Math. 36(2), 239-252 (2016)

15. Kumar, S., Kumar, D., Singh, J.: Fractional modelling arising in unidirectional propagation of long waves in dispersive media. Adv. Nonlinear Anal. 5(4), 383-394 (2016)

16. Molica Bisci, J., Repovs, D.: Multiple solutions of $p$-biharmonic equations with Navier boundary conditions. Complex Var. Elliptic Equ. 59(2), 271-284 (2014)

17. Consiglieri, L.: Radiative effects for some bidimensional thermoelectric problems. Adv. Nonlinear Anal. 5(4), 347-366 (2016)

18. Khader, M.M.: On the numerical solution and convergence study for system of non-linear fractional diffusion equations. Can. J. Phys. 92(12), 1658-1666 (2014)

19. Khader, M.M., Hendy, A.S.: A new Chebyshev computational matrix method: an application for solving the high order fractional differential equations. Walailak J. Sci. Technol. 11(4), 289-305 (2014)

20. Khader, M.M.: Shifted Chebyshev collocation method for the flow and heat transfer due to a stretching sheet embedded in a porous medium with variable thickness, variable thermal conductivity and thermal radiation. Mediterr. J. Math. 13(4), 2319-2336 (2015)

21. Wang, C.: Analytic solutions for a liquid thin film on an unsteady stretching surface. Heat Mass Transf. 42, 759-766 (2006)

22. Mason, J.C., Handscomb, D.C.: Chebyshev Polynomials. Chapman \& Hall, New York (2003) 
23. Sweilam, N.H., Nagy, A.M., El-Sayed, A.: On the numerical solution of space fractional order diffusion equation via shifted Chebyshev polynomials of the third kind. J. King Saud Univ., Sci. 28, 41-47 (2016)

24. Handan, C.Y.: Numerical solution of fractional Riccati differential equation via shifted Chebyshev polynomials of the third kind. J. Eng. Technol. Appl. Sci. 28, 1-11 (2017)

25. Noor, N.F.M., Hashim, I.: Thermocapillarity and magnetic field effects in a thin liquid film on an unsteady stretching surface. Int. J. Heat Mass Transf. 53, 2044-2051 (2010)

Submit your manuscript to a SpringerOpen ${ }^{\circ}$ journal and benefit from:

- Convenient online submission

- Rigorous peer review

- Open access: articles freely available online

- High visibility within the field

- Retaining the copyright to your article

Submit your next manuscript at $\gg$ springeropen.com 